\title{
The impact of inlet flow conditions on the aerodynamic performance of a NACA submerged intake for ground vehicle applications.
}

\author{
J C Holt, K P Garry \\ Applied Aerodynamics Group, School of Engineering \\ Cranfield University, Bedford UK
}

\begin{abstract}
Results are presented following a series of experimental measurements on a submerged NACA type intake orientated between \pm 30 degrees yaw to the free stream in an atmospheric boundary layer wind tunnel at a unit Reynolds number of nominally $1 \times 10^{6}$. The intake was subjected to a range of upstream wall boundary layer conditions and the intake mass flow (as measured by an orifice plate) was monitored to assess aerodynamic performance. The mass flow data is supported by qualitative flow visualisation within the duct, using a smoke filament illuminated in a laser light sheet in order to gain insight into the flow physics.

Intake performance, expressed in terms of a non-dimensional flow momentum coefficient, is seen to degrade with both: (i) intake orientation to the free stream - changes of nominally $40 \%$ are seen for the angle range tested and (ii) increase in upstream boundary layer displacement thickness - changes of nominally $30 \%$ are seen for the range tested. This data is presented as a graphical carpet plot, it is intended that this be used as a guide to performance prediction in non-aeronautical applications where there are often significant changes in both local flow direction and boundary layer thickness. Flow visualisation studies show that the intake performance degradation with yaw angle can be attributed to a progressive change in the vortex- pair structure within the intake as the local flow angle is increased. An increase in both lateral separation and size of the respective vortex cores is considered to act so as to reduce the magnitude of the induced inflow into the intake.
\end{abstract}


Keywords: Flush intake, NACA intake, Submerged intake, Automotive Cooling, Intake effectiveness

\section{Background}

There is considerable interest in the use of flush mounted intakes for aerodynamic cooling in automotive applications. The advantages of this type of intake in respect of a relatively low external drag penalty, greater flexibility in terms of location on the body together with an aesthetic appearance, has resulted in such configurations being adopted in a range of production vehicles. Between 1945 and 1951 the National Advisory Committee for Aeronautics (NACA) published a series of Memoranda and Technical Notes [see for example: Delany (1), Mossman et al (3) and Martin et al (2)] that detail the aerodynamic design of a generic submerged intake suitable for aerospace applications. This arrangement is now colloquially referred to as a 'NACA Intake' and it is to this specification that many vehicle aerodynamicists have turned when seeking to exploit the advantages of flush intakes in automotive applications.

By its nature, a flush mounted intake is more susceptible to changes in upstream boundary layer thickness and inlet flow angle than a conventional annular intake for example. As a result information is needed about the likely impact of such effects on intake performance (assessed in terms of intake mass flow) when extrapolating design guidelines optimised for aeronautical applications to a ground vehicle environment. Relatively little previous work has been carried out in this area and the majority is targeted at aerospace applications in which upstream boundary layers will be relatively thin and relative yaw angles to the free stream small. 
Goetz (4) refers to measurements made over a limited range of intake orientations to the free stream and suggests that there is a potential degradation. More recently, a CFD study backed by experimental data presented by Shütz (5) shows significant reductions in the intake pressure recovery with increase in the boundary layer thickness at the intake inlet and smaller, but still significant, reductions with cross flow (or yaw) angle.

There is a need for detailed data related to intake performance under the conditions typically experienced in automotive applications. This is particularly important if these automotive applications are to include under-body and rear-body intake locations at which local boundary layer thickness will be large and relative yaw angles to the free stream potentially high. In addition, an improved understanding of the aerodynamic flow structure within the intake duct and the manner in which it changes with inlet flow variables would be of considerable benefit when interpreting CFD simulations. A series of measurements were therefore undertaken in which a typical NACA submerged intake configuration is subjected to a range of both inlet boundary layer conditions and simulated cross flow angles, in a controlled wind tunnel environment. Measurements of intake mass flow and flow visualisation of the vortex structure in the duct were used to assess the impact of these two variables and suggest empirical 'corrections' that could be used when attempting intake performance predictions in relatively complex flow environments.

\section{Experimental methodology and design}

An experimental approach was adopted in which a generic NACA intake was manufactured and mounted flush to the working section floor of the Cranfield University, Atmospheric Boundary Layer Wind Tunnel (ABLWT). Modification of the surface roughness on the fetch upstream of the working 
section made it possible to vary the thickness of the floor boundary layer at the intake location. In addition, the intake was mounted in an adjustable turntable so that the intake longitudinal axis could be varied relative to the free stream flow direction, simulating cross flow conditions in the range $-30<\beta<30$ degrees.

The ABLWT has a rectangular working section $2.4 \mathrm{~m}$ wide and $1.2 \mathrm{~m}$ high, positioned at the end of a $13 \mathrm{~m}$ long flow development section. A variable speed DC motor driving a fixed pitch 5 blade fan makes it possible to generate flow velocities in the working section in the range $0.5<U_{\infty}<17 \mathrm{~m} / \mathrm{s}$ at nominally atmospheric temperature and pressure.

The geometry of the NACA intake used throughout this investigation was designed according to the guidelines given by Mossman et al. (3) , in this instance an intake ramp angle of 7 degrees was selected, with a straight ramp profile, see Figures $1(a)$ and $1(b)$. This particular profile was chosen as it is known to perform well under the conditions likely to be experienced by passenger cars. The geometry of the intake lip is known to affect the aerodynamic performance of the intake. The profile adopted in this application is shown in Figure 1(c).

The downstream end of the intake is connected to a tapered diffuser which blends the rectangular cross sectional profile at the intake exit to a cylindrical semi-flexible pipe with an internal diameter of $100 \mathrm{~mm}$, over a distance of $300 \mathrm{~mm}$. see Figure $1(\mathrm{a})$. The NACA intake is embedded in a turntable flush with the working section floor for the three deepest boundary layer profiles (designated BL3, BL4 and BL5 in Table 1), see Figure 2(a). In order to create the two thinnest boundary layer profiles (designated BL1 and BL2 in Table 1) the turntable is installed into a $12 \mathrm{~mm}$ deep splitter plate mounted $230 \mathrm{~mm}$ above the working section floor, see Figure 2(b). 
In practice the operating Reynolds number of this type of intake on a passenger car will vary considerably. Experience suggests that intakes varying in overall length from $80 \mathrm{~mm}$ to $400 \mathrm{~mm}$ are used and that they will be expected to operate at speeds in the range of $15-35 \mathrm{~m} / \mathrm{s}$. This equates to an intake Reynolds number in the range $8200<\operatorname{Re}_{\text {int }}<96,300$. The wind tunnel tests were conducted near the middle of this range at nominally $\mathrm{Re}_{\mathrm{int}}=55,000$.

Similarly, the local boundary layer thickness likely to be experienced by an intake on a typical passenger car will vary considerably depending on location on the vehicle. Estimates of local boundary layer thickness $(\delta)$ for typical passenger vehicle locations give the range as $2 \mathrm{~mm}<\delta<250 \mathrm{~mm}$. Using the throat size $\left(z_{1}\right)$ to non-dimensionalise the size of the intake, this corresponds to a range of $0.25<\delta / z_{1}<$ 6.09. The values of $\delta / z_{1}$ used in the wind tunnel study, given in Table 1 , are in a similar range $0.41<\delta / z_{1}$ $<7.03$ and it is hoped therefore that the wind tunnel simulation will emulate the conditions likely to be experienced by a passenger vehicle on the road.

\subsection{Instrumentation}

The air drawn into the intake is allowed to exhaust to atmosphere through the short diffuser and cylindrical, semi-flexible, pipe. The pressure difference between the wind tunnel working section and atmosphere (expressed as a pressure coefficient $\left(c_{p}\right)$ based on the free stream velocity $\left(U_{\infty}\right)$ ) is nominally 0.16. The intake mass flow is measured by a bespoke orifice plate in the $100 \mathrm{~mm}$ diameter pipe at a location $1 \mathrm{~m}$ downstream of the NACA intake. The detailed geometry of the orifice plate is given in Figure 3. Surface static pressure tappings are located either side of the orifice flange plate, such that the pressure drop across the plate may be measured with a differential pressure transducer. Calibration of the orifice plate was achieved by metering the air flow from a high pressure source into the pipework upstream of the orifice plate through a plenum chamber using a purpose built cap. Flow metering was by a rotary flow meter with a maximum range of $4000 \mathrm{lpm}$ and measurement accuracy of $+/-201 \mathrm{pm}$. 
The flow could be restricted by a gate valve, allowing a range of steady-state flow conditions from 500 to $4000 \mathrm{lpm}$ to be established while the pressure drop across the orifice plate was recorded. The resulting measurement accuracy for momentum coefficient $\left(c_{\mu}\right)$, taking into account the pressure based instrumentation used in the orifice plate, can be estimated at $+/-0.0035$.

Visualisation of the flow structure within the intake itself is achieved by forming a laser light sheet, arranged such that it is orthogonal to the intake longitudinal axis in a plane just upstream of the intake lip. Smoke particles generated by a Compact Viscount 1400 smoke generator are released into the boundary layer upstream of the working section. The resulting smoke filament is ingested into the intake and the flow field observed using a miniature CCD camera (diameter $11 \mathrm{~mm}$ ) mounted in the short diffuser section downstream of the NACA intake exit, looking upstream. The camera output is recorded digitally at 35 frames/second, for a period of 30 seconds. The images presented in Figure 8 are single frames judged to be representative of the 'mean' flowfield.

\subsection{Upstream Wall Boundary Layer Conditions}

Variations in the upstream wall boundary layer conditions were achieved by adjusting the surface roughness characteristics in the flow development section of the wind tunnel (upstream of the working section). A summary of these conditions is given in Table 1. The corresponding vertical profiles of local velocity $(u)$ and longitudinal turbulence intensity $\left(\sqrt{\overline{u^{\prime 2}}} / U_{\infty}\right)$ are given in Figures $4(\mathrm{a})$ and 4 (b) respectively. These illustrate in each case a characteristic turbulent boundary layer profile, with the possible exception of the thinnest boundary layer (BL1), for which there was relatively little data. 


\begin{tabular}{|c|c|c|c|c|c|}
\hline & $\delta(\mathrm{mm})$ & $\delta^{*}(\mathrm{~mm})$ & $\begin{array}{c}\mathrm{Re} / \mathrm{m} \\
\text { (based on free } \\
\text { stream velocity, } \mathrm{U}_{\infty} \text { ) }\end{array}$ & $\delta / z_{1}$ & $\begin{array}{l}\text { details of the roughness } \\
\text { configuration used in the flow } \\
\text { development section }\end{array}$ \\
\hline BL 1 & 23 & 4.9 & $1.03 \times 10^{6}$ & 0.41 & clean splitter plate (see Figure $2 b$ ) \\
\hline BL 2 & 52 & 7.5 & $0.98 \times 10^{6}$ & 0.94 & $\begin{array}{l}\text { splitter plate with } 6 \mathrm{~mm} \text { boundary } \\
\text { layer trip }\end{array}$ \\
\hline BL 3 & 190 & 27.3 & $1.00 \times 10^{6}$ & 3.42 & $\begin{array}{l}\text { clean ABLWT flow development } \\
\text { section. }\end{array}$ \\
\hline BL 4 & 400 & 43.5 & $1.03 \times 10^{6}$ & 7.21 & $\begin{array}{l}\text { distributed } 100 \mathrm{~mm} \text { cube shaped } \\
\text { roughness elements in a diamond } \\
\text { pattern extending } 5 \mathrm{~m} \text { upstream of } \\
\text { the working section. } \\
\end{array}$ \\
\hline BL 5 & 390 & 50.8 & $1.05 \times 10^{6}$ & 7.03 & $\begin{array}{l}\text { distributed } 100 \mathrm{~mm} \text { cube shaped } \\
\text { roughness elements in a diamond } \\
\text { pattern extending } 8 \mathrm{~m} \text { upstream of } \\
\text { the working section. }\end{array}$ \\
\hline
\end{tabular}

Table 1. A summary of the upstream boundary layer characteristics used in the test programme. $\left(z_{1}\right.$ is the intake depth at the lip $(55.5 \mathrm{~mm})$, see Figure 1(a))

\subsection{Influence of inlet boundary layer characteristics on intake performance.}

The intake flow, measured by the orifice plate, is expressed as a non-dimensional mass flow coefficient $\left(c_{\mu}\right)$ based on the measured free stream conditions $\left(\frac{1}{2} \rho U_{\infty}^{2}\right)$, the intake cross sectional area at the throat

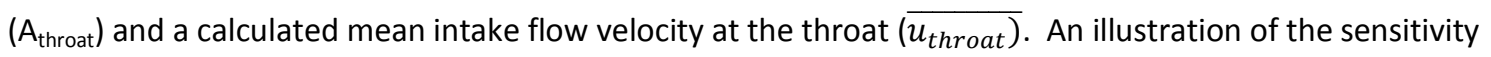
of the intake to changes in wall boundary layer thickness at three angles of orientation to the free stream, is shown in Figure 6. The intake mass flow (at 0,16 and 30 degrees yaw) is seen to marginally increase with reducing boundary layer displacement thickness $\left(\delta^{*}\right)$ until $7.5 \mathrm{~mm}<\delta^{*}<5 \mathrm{~mm}$ where there is a more significant increase for the 16 and 30 degree yaw cases. This may indicate a 'critical' upstream wall boundary layer thickness for this intake at low yaw angles ( $\beta<16$ degrees).

An indication of the effect of Reynolds number changes is given in Figure 5 which presents the variation of intake flow rate with wind tunnel speed at different intake yaw angles for a fixed upstream roughness condition corresponding to BL3. The data suggests that there are no significant consequences of changes 
in Reynolds number, within the range tested. Clearly the vertical flow structure within the intake would be expected to be sensitive to Reynolds number at the extremes of the range considered but it is not possible to establish the impact of this from the current data set.

A more detailed assessment of the response of the intake to changes in yaw angle relative to the free stream is given in Figure 7. In general, the intake flow reduces with increasing yaw angle in the range tested $(+30<\beta<-30$ degrees). The thicker the upstream boundary layer the more progressive the degradation with increasing yaw angle. For the thinner boundary layers the majority of the degradation occurred at yaw angles ( $\beta<8$ degrees).

To aid comparison, the intake flow data in Figure 7 have been normalised using the hypothetical 'maximum possible' flow rate - corresponding to capture of $100 \%$ of the free stream by the intake for the thinnest boundary layer case $\left(B L 1, \delta^{*}=4.9 \mathrm{~mm}\right)$. The resulting degradation in the maximum flow rate (which is obtained at zero yaw in each case) corresponds to nominally $10 \%$ of the theoretical maximum for the range of boundary layer thicknesses tested.

\subsection{Intake Vortex Flow Visualisation.}

An indication of the change in the intake flow characteristics with orientation of the longitudinal axis to the free stream, can be obtained from the light sheet illumination of a smoke filament entrained into the intake over a range of intake yaw angles, given in Figures $8(a)$ to (j). The image plane is fixed relative to the intake longitudinal axis, the leeward side to the right, windward to the left. The location of the core of the vortices generated within the intake can be identified by the clear region at the centre of the entrained smoke filament. 
A qualitative indication of the degree to which the vortex has diffused can be made by estimating the size of the concentrated smoke region associated with each vortex structure. In the case of the leeward vortex, the size of the recirculation region is seen to progressively increase with increasing intake yaw angle from nominally $15 \%$ of the intake cross sectional area at zero yaw to more than $50 \%$ at 30 degrees yaw. It is thought that this increase is size will result in a reduction of the induced downwash velocity. This is consistent with the reduction in the measured intake mass flow with increasing yaw angle.

In addition to the size of the vortex the relative locations of the two windward and leeward vortex structures will impact on the magnitude of the induced downwash. An illustration of the vortex trajectory with varying intake orientation angle, derived from inspection of the flow visualisation photographs, is given in Figure 10. It can be seen that the vortices that form with the intake at zero yaw (the optimum 'design' condition) are positioned close to the intake side wall. The vortex that is formed on what becomes the leeward edge of the intake, migrates progressively towards the intake centre line with increasing yaw angle, but remains at nominally the same vertical position relative to the lip. In contrast the windward vortex is relatively short lived, weakening rapidly and becoming difficult to identify at yaw angles greater than 10 degrees. The lateral position of the windward vortex relative to the intake lip remains nominally constant but it migrates vertically.

The change in size of the leeward vortex with increasing intake orientation to the free stream is illustrated in Figure 9. This data, derived from inspection of the flow visualisation photographs, shows how the dominant vortex structure occupies nominally $15 \%$ of the intake cross sectional area at zero degrees yaw and expands in a fairly predictable manner until it occupies nominally $55 \%$ of the duct area at 30 degrees yaw. This growth or diffusion in the vortex will be related to the induced downwash 
velocity and account for a proportion of the degradation of intake effectiveness with increasing yaw angle.

It is recognised that the magnitude of the vortex and possibly the vortex trajectory is likely to be sensitive to Reynolds number. These qualitative data are therefore given as an aid to the interpretation of CFD simulations and an indication of trends.

A summary of the performance of the intake under different upstream boundary layer conditions and orientations relative to the free steam is given in Figure 11. The non-dimensional intake flow momentum coefficient can be used as an indicator of intake effectiveness and it is seen to be most sensitive to intake orientation to the free stream for moderate upstream boundary layer thicknesses $\left(0.14<\delta^{*} / z_{1}<0.78\right)$. There is a marked improvement in intake effectiveness when the orientation angle is small and the upstream boundary layer is thin $\left(\delta^{*} / z_{1}<0.14\right)$. This is consistent with the flow conditions and applications for which this type of intake was designed, but represents inlet conditions that are unlikely to be encountered in conventional automotive applications. Established intake flow prediction data should therefore be used with caution and a correction consistent with the data presented in Figure 11 considered.

\section{Conclusions.}

The aerodynamic effectiveness of flush mounted intakes can be assessed in terms of a non-dimensional intake flow momentum coefficient $\left(c_{\mu}\right)$. A series of measurements within an up-scaled boundary layer on a generic NACA type intake are used to show that the effectiveness decreases as the angle between the intake longitudinal axis and the free stream flow direction is increased. This can be attributed to a reduction in the induced 'downwash' velocity component into the intake as a result of the formation of 
the contra-rotating vortex pair above the intake ramp. Increasing orientation angle causes the leeward edge vortex to expand and the windward edge vortex to diminish. Intake effectiveness increases as the thickness of the boundary layer upstream of the intake is reduced. In order to relate these two effects the data is presented in a carpet plot which may facilitate prediction of the intake performance changes for typical ground vehicle applications in which both (a) the local flow angle will be varying and off the intake axis and (b) the local boundary layer thickness upstream of the intake will be much higher than in the aerospace applications for which this type of intake was originally designed.

\section{Acknowledgements}

This research received no specific grant from any funding agency in the public, commercial, or not-forprofit sectors. A preliminary investigation which provided a useful platform for the current work was conducted by Benjamin Goffette (6) in his 2009 MSc Thesis.

\section{References}

1. Delany, N.K. An investigation of submerged air inlets on a $1 / 4$-scale model of a typical fightertype airplane. NACA RM A8A20 (1948).

2. Martin, N.J. Holzhauser, C.A. An experimental investigation at large scale of several configurations of an NACA submerged air intake. NACA RM A8F21 (1948).

3. Mossman, E. Randall, L. An experimental investigation of the design variables for NACA submerged duct entrances. NACA Research Memorandum A7130 (1948). 
4. Goetz, H. The influence of wind tunnel tests on body design, ventilation and surface deposits of sedans and sports cars. SAE Automotive Engineering Congress, Detroit Michigan. (1971)

5. Schütz, T. Numerische Studie von NACA-Lüfteinlässen an Kraftfahrzeugen. Diplomarbeit Institut für Verbrennungsmotoren und Kraftfahrwesen, Universität Stuttgart (2005).

6. Goffette, B. Experimental investigation and modelling of the effects of yaw and inlet boundary layer profile on the performance of the NACA duct. Cranfield University, MSc Thesis (2009)

Notation \& Abbreviations

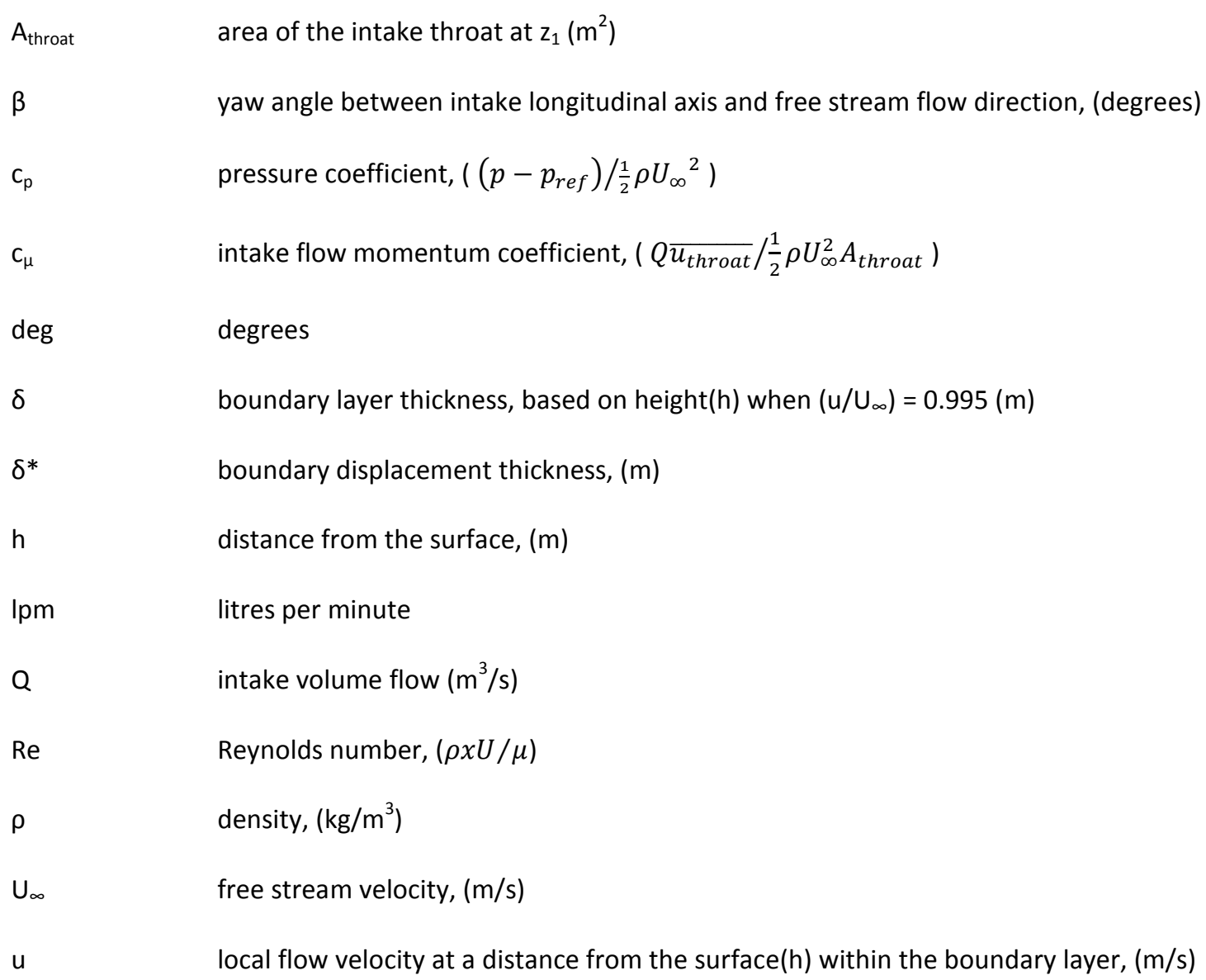


fluctuating local flow velocity, $(\mathrm{m} / \mathrm{s})$

$\mathrm{u}_{\text {throat }}$

flow velocity at the intake throat $(\mathrm{m} / \mathrm{s})$

$\mathrm{z}_{1}$

Inlet height measured in inlet plane relative to ramp floor $(\mathrm{m})$ 


\begin{tabular}{|c|c|c|c|}
\hline x (upper) & y (upper) & $\mathrm{x}$ (lower) & y (lower) \\
\hline 0 & 0 & 33.2 & -8.4 \\
\hline 0.2 & 1.13 & 32.2 & -8.37 \\
\hline 1.2 & 2.61 & 31.2 & -8.27 \\
\hline 2.2 & 3.55 & 30.2 & -8.27 \\
\hline 3.2 & 4.31 & 29.2 & -8.27 \\
\hline 4.2 & 4.96 & 28.2 & -8.22 \\
\hline 5.2 & 5.54 & 27.2 & -8.18 \\
\hline 6.2 & 6.06 & 26.2 & -8.12 \\
\hline 7.2 & 6.52 & 25.2 & -8.06 \\
\hline 8.2 & 6.93 & 24.2 & -7.98 \\
\hline 9.2 & 7.29 & 23.2 & -7.9 \\
\hline 10.2 & 7.64 & 22.2 & -7.81 \\
\hline 11.2 & 7.97 & 21.2 & -7.71 \\
\hline 12.2 & 8.29 & 20.2 & -7.59 \\
\hline 13.2 & 8.61 & 19.2 & -7.46 \\
\hline 14.2 & 8.92 & 18.2 & -7.33 \\
\hline 15.2 & 9.21 & 17.2 & -7.19 \\
\hline 16.2 & 9.49 & 16.2 & -7.04 \\
\hline 17.2 & 9.75 & 15.2 & -6.89 \\
\hline 18.2 & 9.99 & 14.2 & -6.73 \\
\hline 19.2 & 10.22 & 13.2 & -6.57 \\
\hline 20.2 & 10.43 & 12.2 & -6.4 \\
\hline 21.2 & 10.63 & 11.2 & -6.23 \\
\hline 22.2 & 10.82 & 10.2 & -6.06 \\
\hline 23.2 & 11.01 & 9.2 & -5.88 \\
\hline 24.2 & 11.2 & 8.2 & -5.66 \\
\hline 25.2 & 11.38 & 7.2 & -5.39 \\
\hline 26.2 & 11.55 & 6.2 & -5.07 \\
\hline 27.2 & 11.72 & 5.2 & -4.67 \\
\hline 28.2 & 11.88 & 4.2 & -4.21 \\
\hline 29.2 & 12.03 & 3.2 & -3.66 \\
\hline 30.2 & 12.18 & 2.2 & -3 \\
\hline 31.2 & 12.32 & 1.2 & -2.16 \\
\hline 32.2 & 12.46 & 0.2 & -0.78 \\
\hline 33.2 & 12.6 & 0 & 0 \\
\hline
\end{tabular}

Table 2. Intake lip coordinates, dimensions in $\mathrm{mm}$ - origin leading edge centre line 


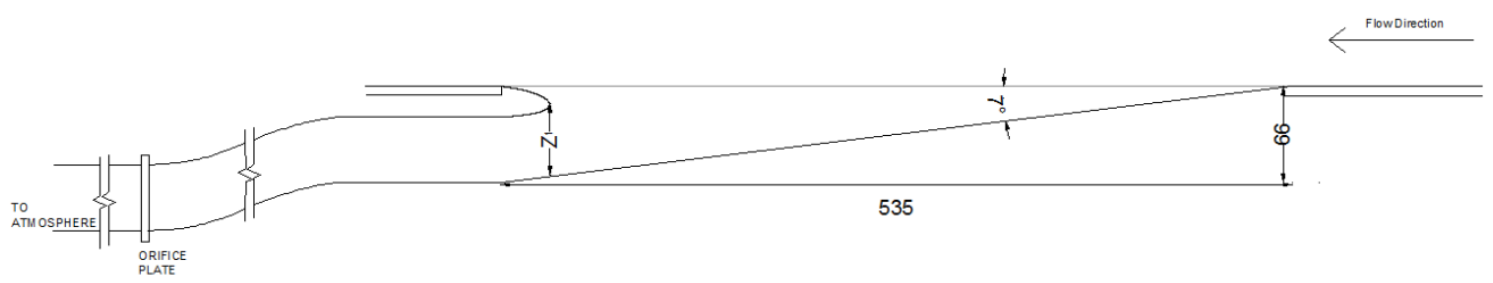

Figure 1(a). Schematic cross-section (on the lateral centre line) of the submerged intake

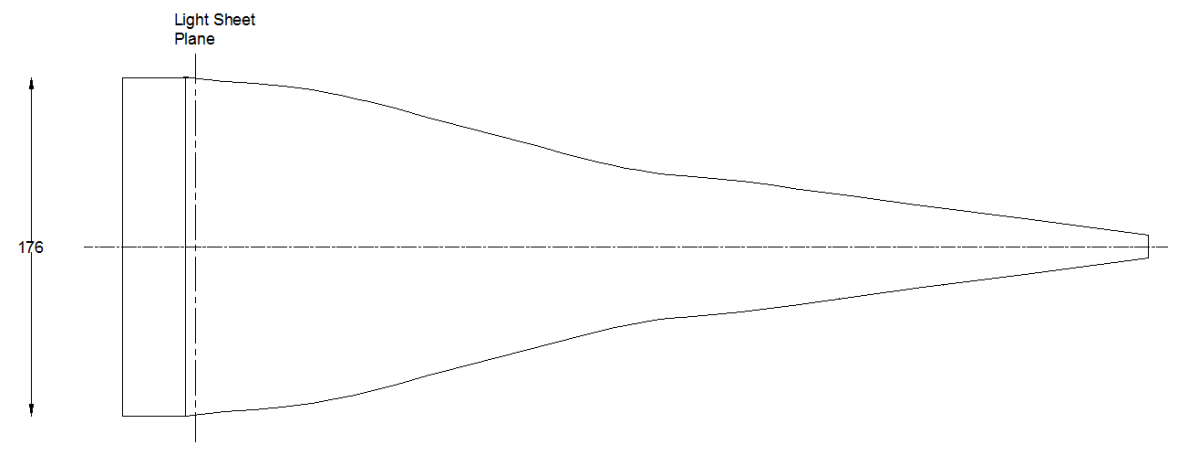

Figure 1(b). NACA intake planform configuration, flow direction from right to left as viewed.

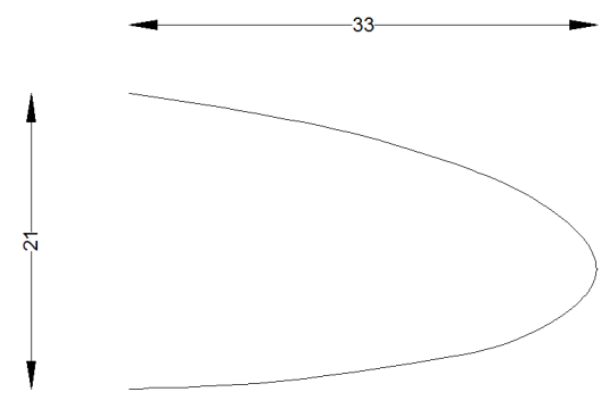

Figure 1(c). Intake lip profile geometry (see co-ordinate details in Table 2) 


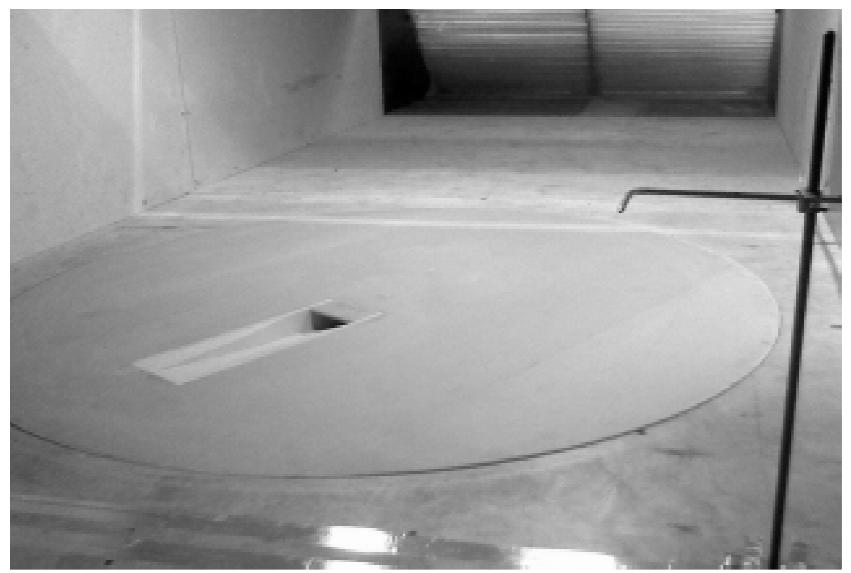

Figure 2a. View, looking downwind, of the ABLWT working section with the NACA intake mounted in the adjustable turntable

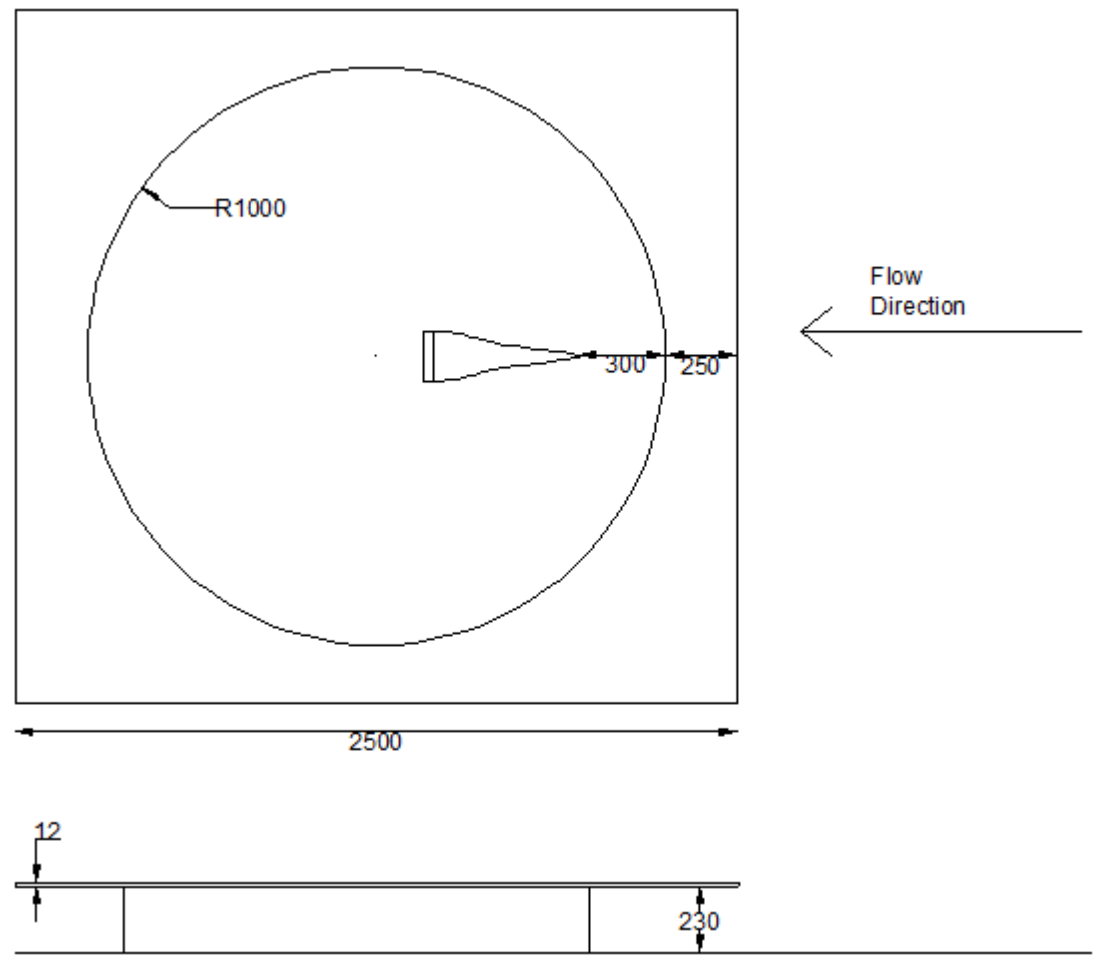


Figure $2 b$. Schematic arrangement of the floor mounted splitter plate used to generate the two thinner

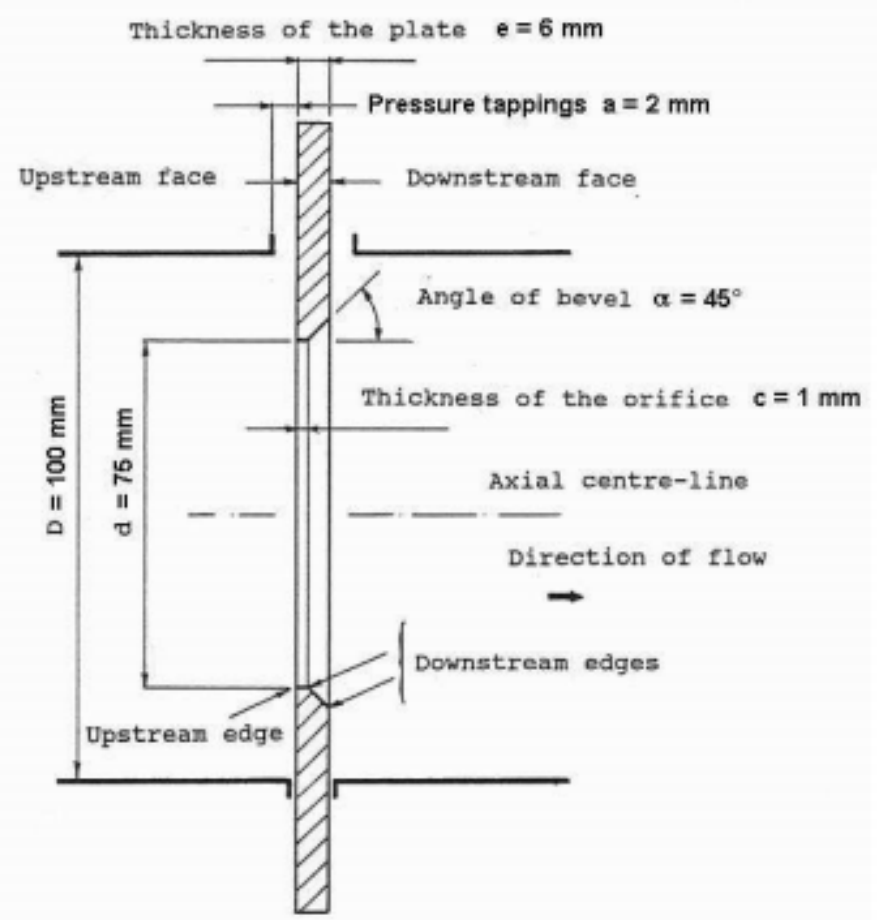

boundary layer profile, $B L 1$ and $B L 2$

Figure 3. Layout and dimensions of the orifice plate used to measure the intake mass flow. 


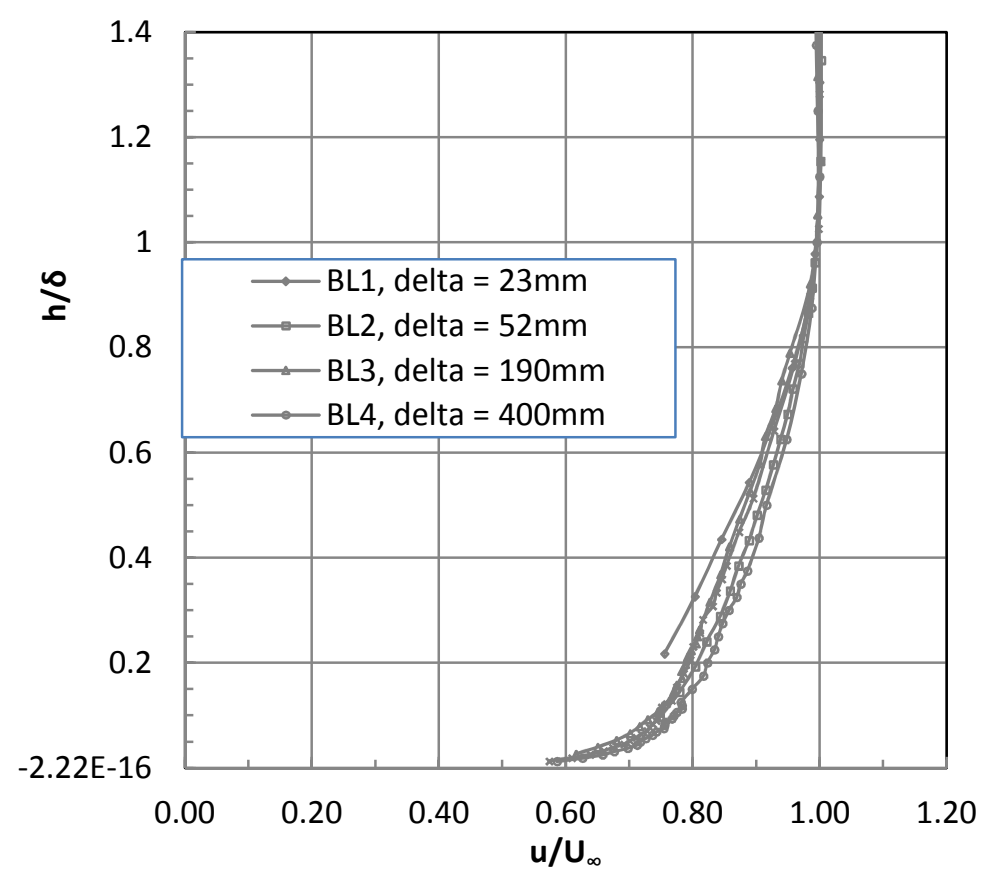

Figure 4a. Non-dimensional boundary layer velocity profiles for the five upstream flow condition cases investigated

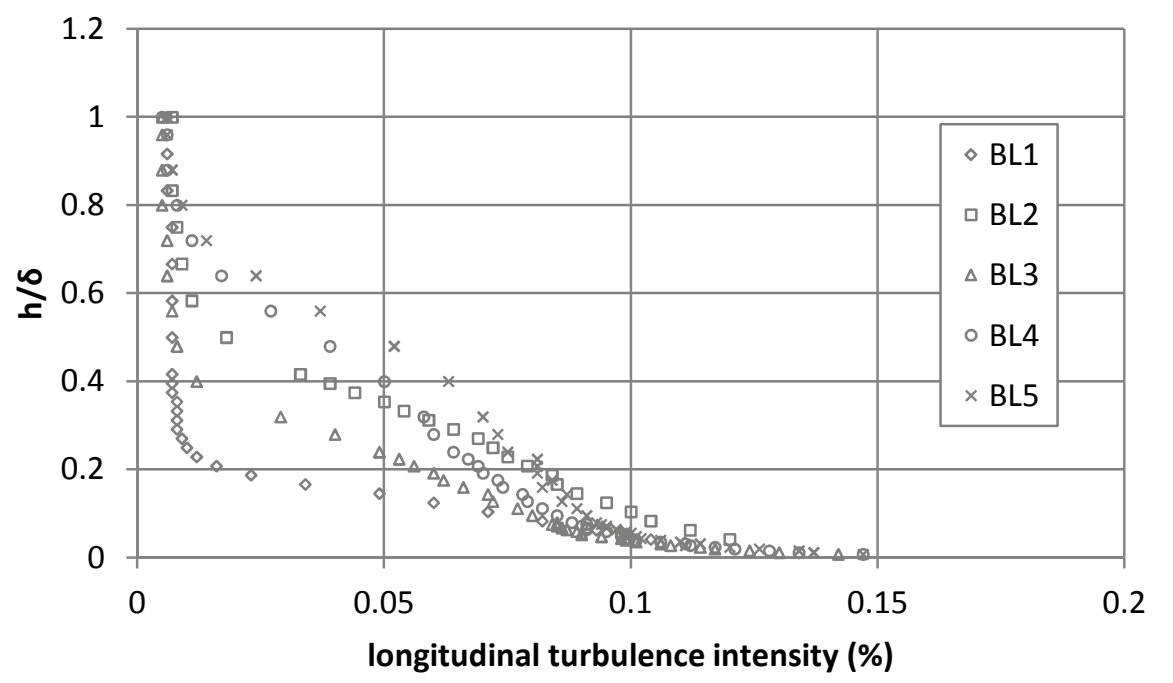

Figure $4 b$. Variation of local turbulence intensity $\left(\sqrt{\overline{u^{\prime 2}}} / U_{\infty}\right)$ with non-dimensional distance from the surface, within each of the five boundary layer cases investigated. 


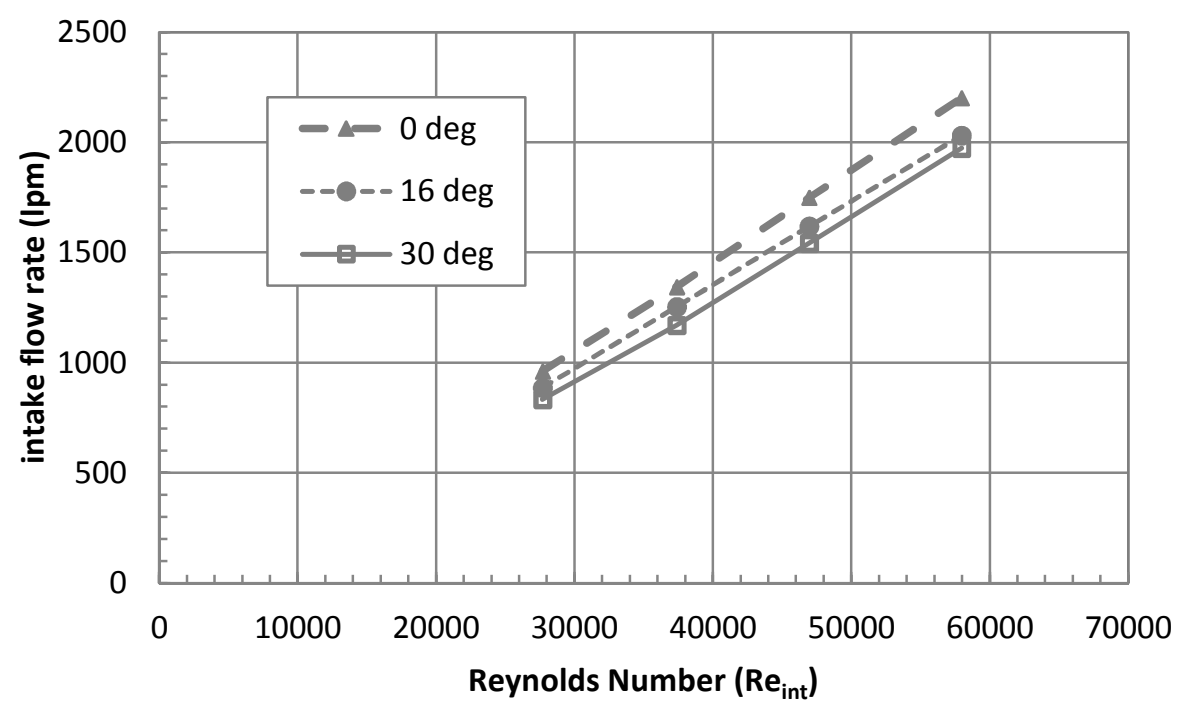

Figure 5. Variation of flow rate with intake Reynolds number for boundary layer profile BL3 


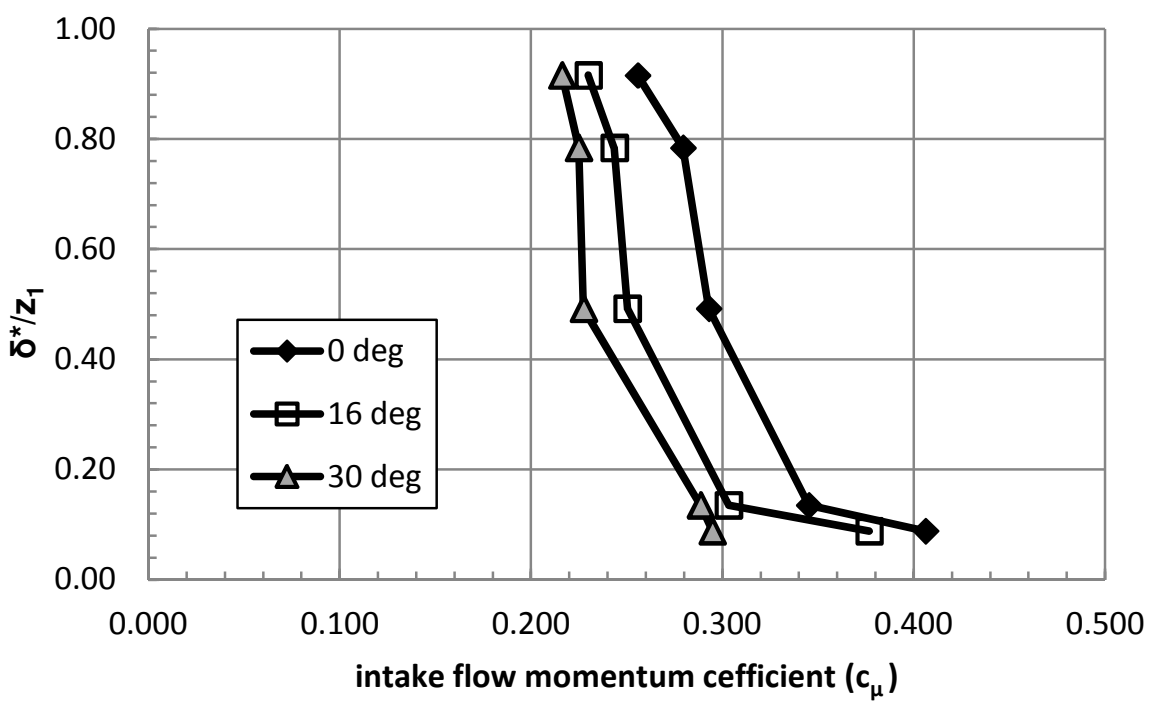

Figure 6. Variation of intake flow Momentum Coefficient $\left(c_{\mu}\right)$ with boundary layer displacement thickness $\left(\delta^{*}\right)$ at 0,16 and 30 degrees yaw. 


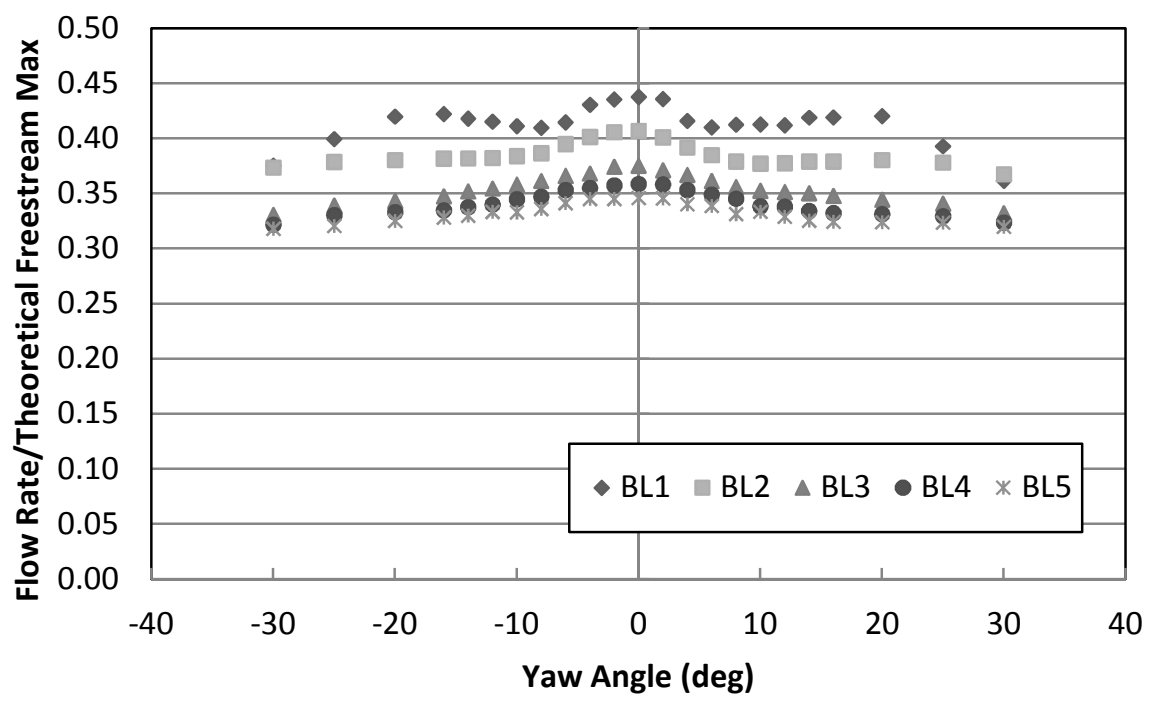

Figure 7. Variation of intake flow rate (normalised by the free stream flow rate through the intake area, $A_{\text {throat }}$ ) with yaw angle for each of the boundary layer conditions considered. 


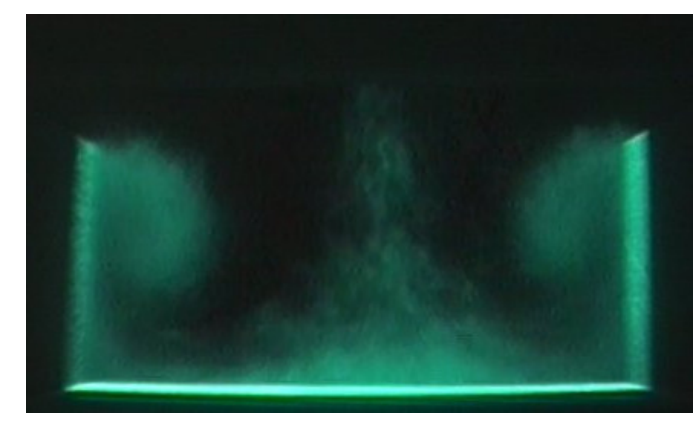

(a) 0 deg Yaw

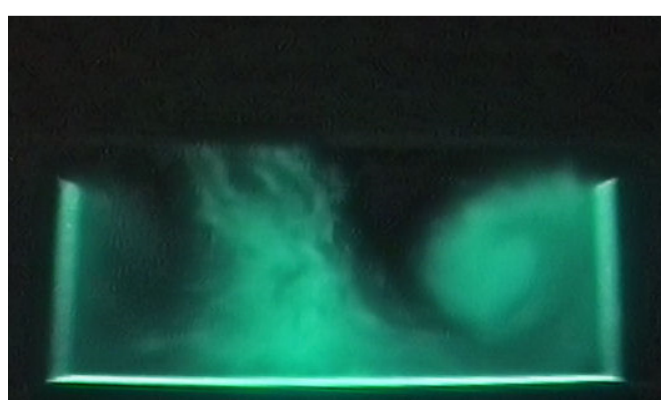

(c) 7 deg yaw

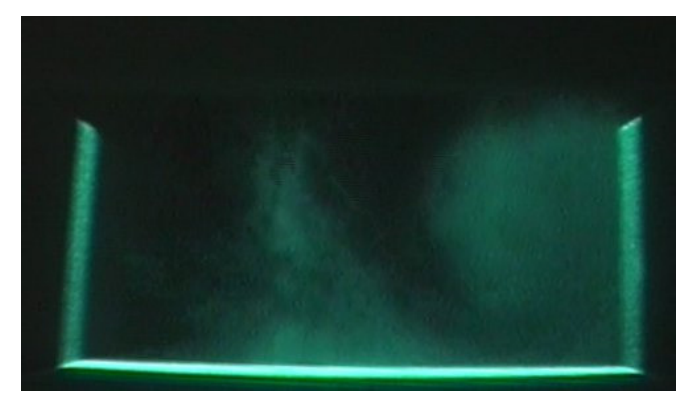

(e) 12 deg yaw

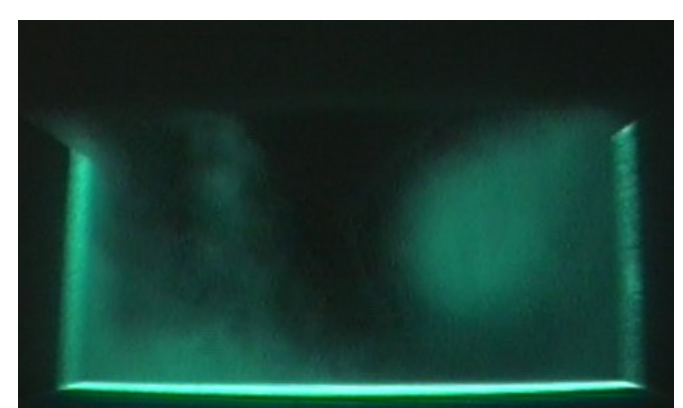

(g) 16 deg Yaw

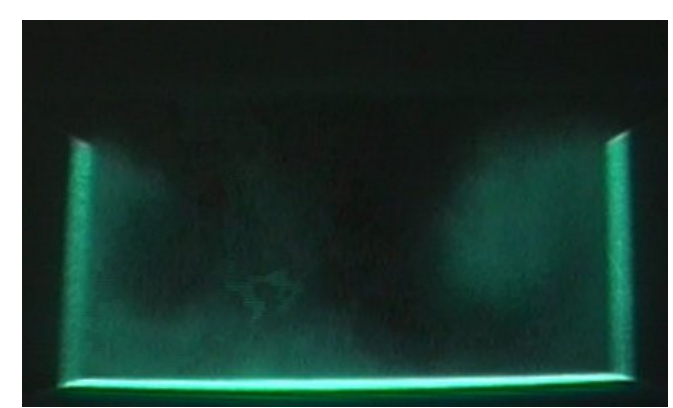

(b) 4 deg yaw

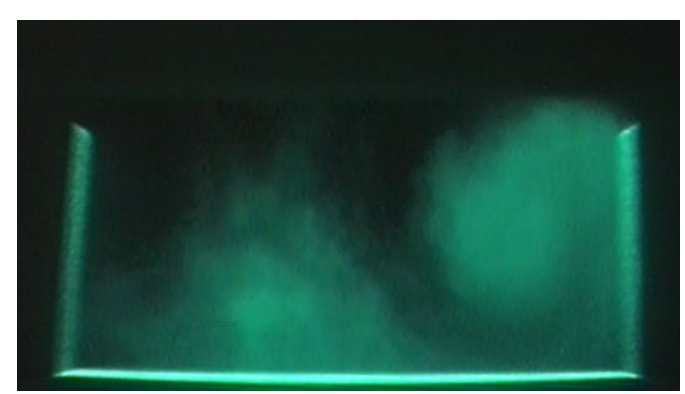

(d) 10 deg Yaw

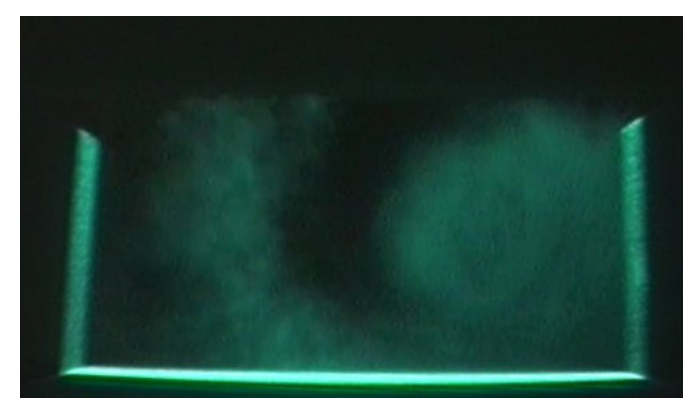

(f) 14 deg yaw

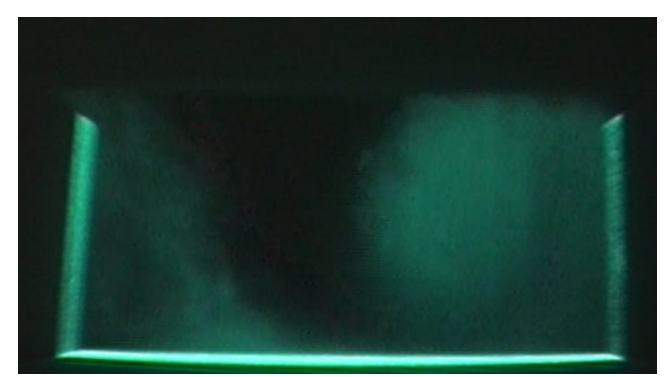

(h) 20 deg Yaw 


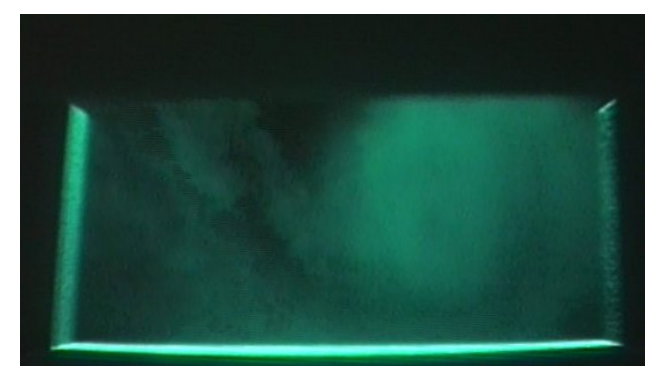

(i) 25 deg Yaw

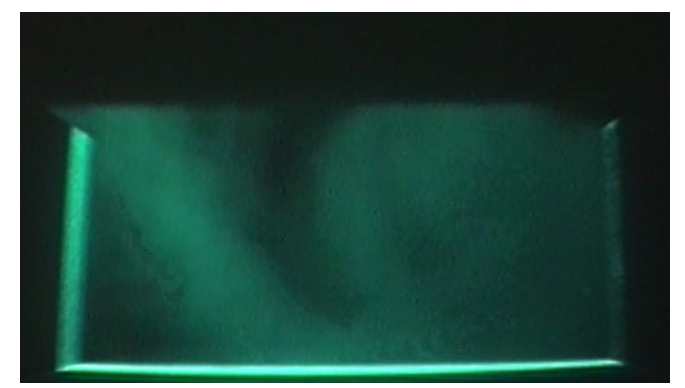

(j) 30 deg yaw

Figure 8. Visualisation of a smoke filament entrained into the intake using a light sheet in a plane, orthogonal to the intake longitudinal axis, at the axial location of the intake lip. View is looking upwind from a position within the intake diffuser. 


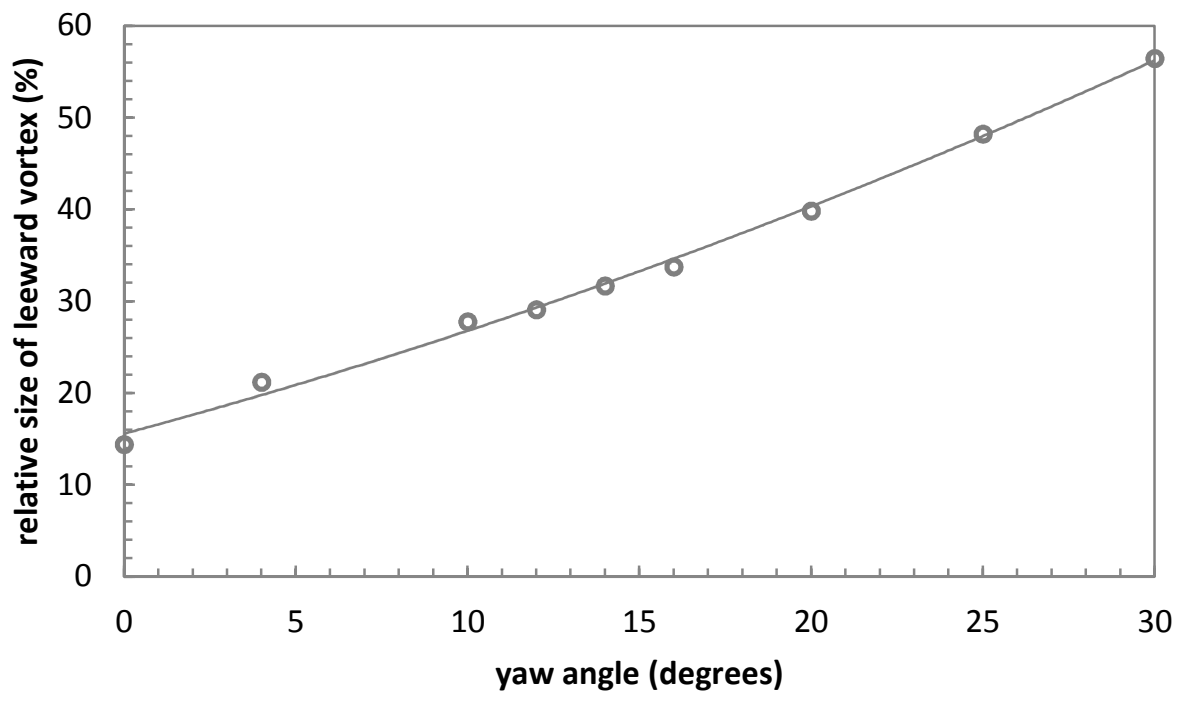

Figure 9. Variation in the size (expressed as a percentage of the intake duct area) of the leeward vortex with yaw angle. 


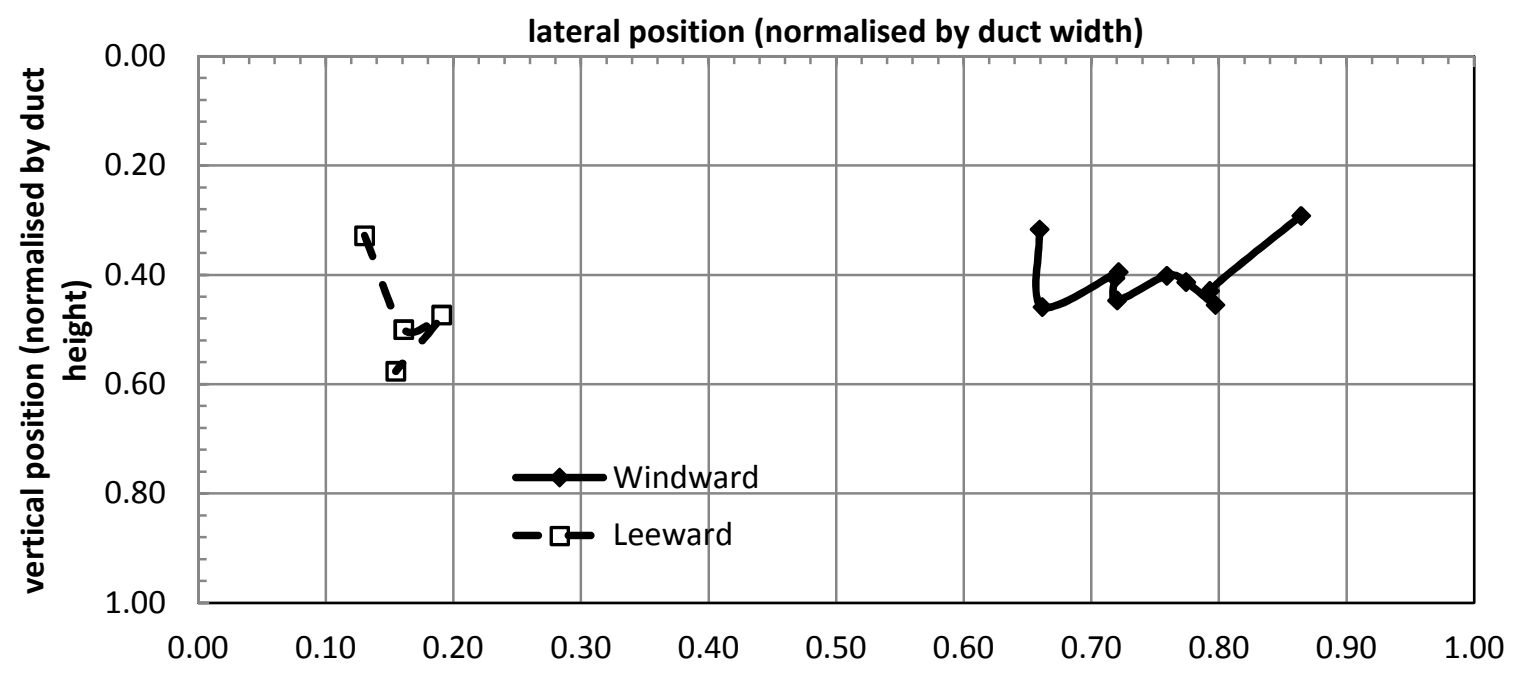

Figure 10. An illustration of the movement and relative location of the windward and leeward vortex centres with increasing intake yaw angle. Corresponds to the plane within the intake illuminated by the light sheet, see Figure 1(b). 


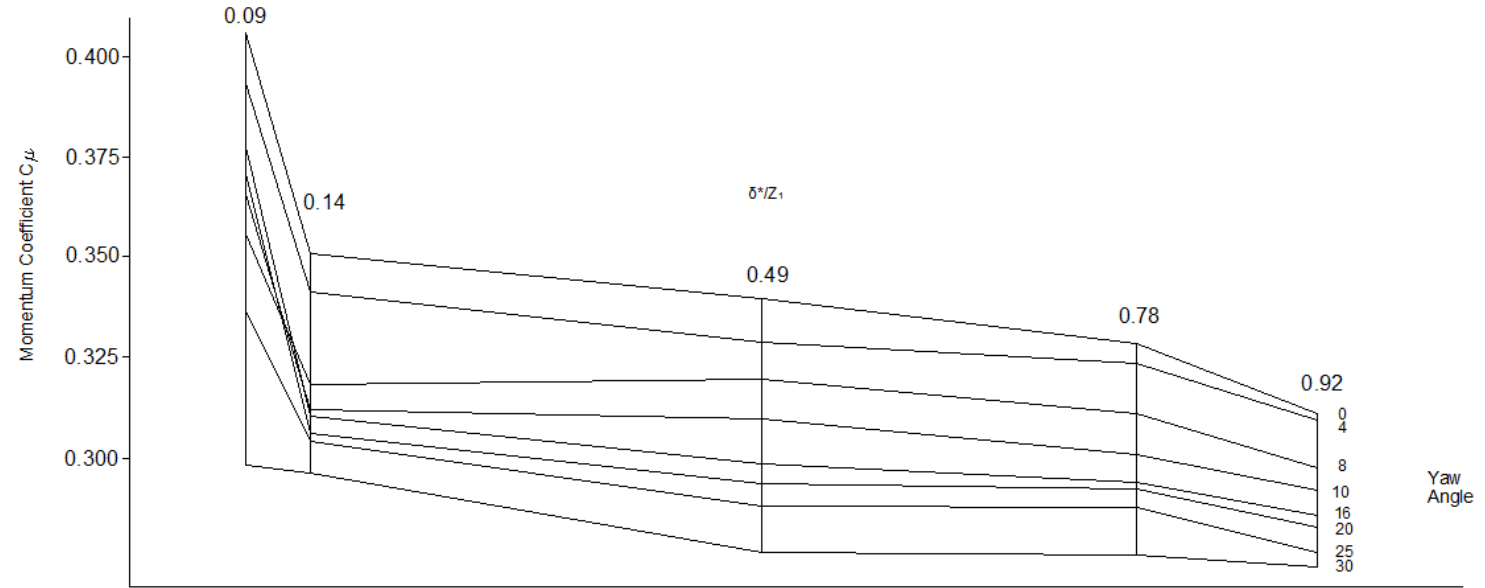

Figure 11. Summary of the effect of both intake orientation angle and upstream boundary layer condition on intake flow momentum coefficient $\left(c_{\mu}\right)$. 